\title{
Ambientes Virtuais de Aprendizagem: Revisão Integrativa de Teses de Doutorado no Contexto Brasileiro entre 2003 e 2012
}

\author{
Paulo Cristiano de Oliveira (CEETEPS-FATEC-ZL/EGC-UFSC, oliveirapco@yahoo.com.br) \\ Marília Kamenschek Carneiro (CEETEPS-FATEC-ZL, mah_mkc@hotmail.com) \\ Shirlene Silva Lopes (CEETEPS-FATEC-ZL, shirlenemm@yahoo.com.br) \\ Marina Keiko Nakayama (EGC-UFSC, marina@egc.ufsc.br)
}

\begin{abstract}
Resumo: Um Ambiente Virtual de Aprendizagem (AVA) é um software que inclui ferramentas para criação, tutoria e gestão das atividades em Educação a Distância (EaD). Este artigo apresenta uma análise das teses de doutorado relacionadas aos AVA, da Biblioteca Digital Brasileira de Teses e Dissertações (BDTD). O método da revisão integrativa orientou a coleta de 58 trabalhos. Levantou-se o número de teses produzidas pelas universidades entre 2003 e 2012, suas áreas temáticas, as palavras-chave utilizadas, os métodos de pesquisa empregados, os AVA utilizados como contexto ou objeto das teses e a aderência às categorias de ferramentas em plataformas educacionais pertinentes a cada um dos estudos. Observou-se que: (1) o método de pesquisa mais empregado nas teses foi o Estudo de Caso, (2) o AVA mais utilizado como contexto de estudo para as teses foi o Moodle e, (3) a maioria dos trabalhos teve como foco o papel do AVA na Coordenação, na Administração e na Comunicação e Interação. O estudo permitiu observar uma tendência de crescimento do número de teses sobre o tema e indicar sugestões para pesquisas futuras com foco na produção de alunos no AVA, na coordenação e comunicação dos AVA e na cooperação e administração dos AVA.
\end{abstract}

Palavras-chave: ambiente virtual de aprendizagem (AVA), revisão integrativa, teses.

\section{Learning Management Systems: Integrative Review of Doctoral theses in the Brazilian Context between 2003 and 2012}

\begin{abstract}
A Learning Management Systems (LMS) is software that includes tools for creating, managing and mentoring activities in Distance Education. This article presents a review of theses about LMS of the Brazilian Digital Library of Theses and Dissertations. The integrative review method guided the collection of 58 theses This research analyzed studies produced by universities between 2003 and 2012, their thematic areas, the keywords used, the research methods employed, LMS used as context or object of the theses and the adherence of the categories of tools in educational platforms related to the jobs analyzed. It was observed that: (1) the research method most often employed in the theses was the Case Study, (2) Moodle was the LMS most used as context of the thesis, and (3) in most researches the focus was about the role of LMS at Coordination, Administration and Communication and Interaction. The study revealed an increasing number of theses about the subject and indicates suggestions for future research focusing on LMS production by students, LMS's coordination and communication and LMS's cooperation and administration.
\end{abstract}

Keywords: learning management systems (LMS), integrative review, theses. 


\section{Introdução}

A Educação a Distância (EaD) pode ser vista como causa e resultado de mudanças significativas na compreensão do conceito de educação, bem como de mudanças de como deve ser organizada (MOORE, KEARSLEY, 2007). A partir da análise da literatura da área de EaD e de trabalhos correlatos (BRITO et al., 2013, CARDOSO, BURNHAM, 2011; ZAWACKI-RICHTER, BÄCKER e SEBASTIANVOGT, 2009), identificou-se que há possíveis lacunas, especialmente de estudos que analisem, de forma transversal, as pesquisas sobre o Ambiente Virtual de Aprendizagem (AVA) na produção científica. Buscando ainda dar subsídios para este estudo, pode-se citar o levantamento de Bach, Domingues e Walter (2013), que apresentou uma revisão sistemática da produção científica brasileira sobre o emprego de Tecnologia da Informação (TI) no ensino entre 1997 e 2011. O estudo apontou que poderiam ser realizadas pesquisas que orientassem as instituições de ensino e os docentes a explorar os recursos que apenas a TI pode oferecer e, consequentemente, melhorar qualitativamente o ensino.

Dessa forma, o objetivo deste estudo é apresentar, em parte, as pesquisas sobre AVA nas teses de doutorado brasileiras, realizando uma revisão integrativa dessas teses no período de 2003 a 2012. O estudo poderá orientar novas pesquisas sobre o tema, pois ao final são apresentadas inferências que podem contribuir para esta área de pesquisa. Os objetivos específicos da pesquisa são: (1) relacionar as teses sobre AVA de acordo com autor, ano de publicação, objetivo e delineamento de pesquisa; (2) identificar os AVA utilizados como contexto para as teses, (3) verificar as principais categorias de ferramentas em plataformas educacionais apresentadas nas teses e, (4) sugerir temáticas para pesquisas sobre AVA, a partir dos resultados da análise.

\section{Fundamentação Teórica}

Promover a EaD não é uma tarefa simples, pois deve-se preocupar não somente com o conteúdo didático, mas também com o gerenciamento e organização de cursos a distância (MOORE, KEARSLEY, 2007). Nesse contexto, há o desenvolvimento dos chamados "sistemas de gerenciamento de aprendizagem" ou LMS (Learning Management System) também conhecidos como AVA. Esses sistemas buscam automatizar a administração de cursos, registrar usuários, cursos, informações sobre o processo e fornecer relatórios para a administração (COUTINHO, 2009).

Há no mercado inúmeras opções de AVA, tanto com licenças sem custo, quanto com licenças pagas, desenvolvidos por empresas privadas, universidades e órgãos do governo. Valente e Matar (2007) indicam que o Blackboard ocupa a primeira posição entre os AVA proprietários e o Moodle, que possui licença gratuita, tem sido cada vez mais utilizado, inclusive no Brasil. Coutinho (2009) aponta que diversos pesquisadores e usuários têm se dedicado a investigar quais são os elementos necessários para a escolha de um AVA. Em 2004, por exemplo, uma equipe do Grupo de Informática, Educação e Sociedade da Universidade Federal do Rio de Janeiro (UFRJ), identificou sete categorias principais de ferramentas em uma plataforma educacional. Essas categorias são: Interface, Navegação, Avaliação, Recursos Didáticos, Comunicação/Interação, Coordenação e Apoio Administrativo. Ressalta-se que essas categorias constituem um suporte para a análise apresentada na seção 3.

Para Roque et al. (2004), a categoria Interface é o elemento através do qual se estabelece a comunicação entre o usuário e o sistema, durante o processo de interação. Deve ser limpa, objetiva, com carregamento rápido, consistente, respeitar o idioma do 
usuário, possibilitar redimensionamento e ter opções de acessibilidade e portabilidade. A categoria Navegação está relacionada com o trânsito livre e fácil entre as páginas de um AVA. Deve ser direta, com padronização de comandos e facilitar a mudança de telas. A categoria Avaliação compreende as formas para determinar se o aluno assimilou de fato o conteúdo proposto. São ferramentas que possibilitam ao professor a avaliação e o acompanhamento do aluno. Devem ser flexíveis e permitir o monitoramento ou rastreamento das atividades. A categoria Recursos Didáticos consiste em ferramentas que o ambiente oferece ao professor. Elas devem ser de fácil utilização e versáteis, permitindo a aplicação e uso pedagógico adequado. A categoria Comunicação/Interação abrange o fluxo de informações entre pessoas no AVA. Os processos de comunicação devem ser mapeados e a interação deve ser estimulada (ROQUE, et al., 2004).

$\mathrm{Na}$ categoria Coordenação concentram-se as atividades de planejamento, criação, execução e controle dos cursos pelo professor. Desta forma, ela deve facilitar a organização dos cursos; possibilitar o acompanhamento do desempenho dos alunos e tutores; e incorporar mecanismos que permitam avaliar a evolução cognitiva dos alunos e definir os papéis dos atores. A categoria Apoio Administrativo reúne as ferramentas de gerência e gestão do ambiente. Seus principais objetos são: integração professor/aluno/curso; produção de relatórios estatísticos; definição de privilégios de acesso; e processamento de inscrições e fornecimento de informações gerais sobre o ambiente (ROQUE, et al., 2004).

Para Gonzales (2005), as funcionalidades dos AVA podem ser organizadas em: (1) de coordenação (servem de suporte para a organização de um curso são utilizadas pelo professor para disponibilizar informações aos alunos, tanto informações das metodologias do curso); (2) de comunicação (englobam fóruns de discussão, bate-papo, correio eletrônico e conferência entre os participantes do ambiente e têm o objetivo de facilitar o processo de ensino-aprendizagem e estimular a colaboração e interação entre os participantes e o aprendizado contínuo); (3) de produção dos alunos (oferecem o espaço de publicação e organização do trabalho dos alunos ou grupos, através do portfólio, diário, mural e perfil); (4) de cooperação e de administração (oferecem recursos de gerenciamento, do curso, dos alunos e de apoio a tutoria). Essas funcionalidades constituirão um apoio para a sugestão de estudos, a partir das lacunas identificadas por esta pesquisa.

\section{Procedimentos Metodológicos}

Para que os objetivos deste estudo pudessem ser alcançados, foi realizada uma revisão integrativa (JACKSON, 1980). A revisão integrativa oferece o acesso rápido aos resultados relevantes de pesquisas que fundamentam as condutas ou a tomada de decisão, proporcionando um saber crítico (JACKSON, 1980, MENDES, SILVEIRA e GALVÃO, 2008). O método da revisão integrativa possui a capacidade de sistematizar o conhecimento científico relacionado à determinada área de conhecimento (WHITTEMORE, KNAFL, 2005, BOTELHO, CUNHA e MACEDO, 2011).

$\mathrm{Na}$ medida em que traça um panorama da produção científica relacionada com um determinado assunto, a revisão integrativa aproxima o pesquisador de problemas a serem estudados (BOTELHO, CUNHA e MACEDO, 2011). Neste contexto, o método possibilita conhecer a evolução de estudos sobre os AVA ao longo do tempo e, com isso, permite que se possa vislumbrar novas possibilidades de pesquisa. Os mesmos autores indicam que a revisão integrativa deve seguir algumas etapas bem definidas que são: (1) identificação do tema e seleção da questão de pesquisa, (2) estabelecimento dos 
critérios de exclusão e inclusão, (3) identificação dos estudos pré-selecionados e selecionados, (4) categorização dos estudos selecionados, (5) análise e interpretação dos resultados e, (6) apresentação da revisão e síntese do conhecimento.

Este estudo consistiu na recuperação e na análise somente de teses, provenientes de instituições de ensino e pesquisa, disponíveis em meio eletrônico, no repositório da Biblioteca Digital Brasileira de Teses e Dissertações - BDTD ${ }^{1}$ (SOUTHWICK, 2003). Trata-se de uma pesquisa exploratória, na medida em que proporciona uma visão geral dos AVA no desenvolvimento de estudos relatados em teses (GIL, 2008). No mapeamento, foi utilizada a "Procura Avançada"2, disponibilizada no site do BDTD. Preencheu-se o campo Assunto com a expressão "ambiente virtual de aprendizagem" (entre aspas) e também “ambientes virtuais de aprendizagem” (entre aspas). Foram utilizadas ainda as seguintes opções: País=Brasil, Grau=Teses, Idioma=Português, Ano de Defesa: a partir de 2002 (a busca não considera o ano de 2002) e/ou até 2013 (a busca não considera o ano de 2013). Antes de realizar a busca que resultou neste artigo, foram feitos testes para que se pudesse conhecer o comportamento da base de dados.

Foram coletadas, inicialmente, 58 teses, porém, oito dessas teses não foram utilizadas na pesquisa, uma vez que numa análise mais detalhada, se percebeu que seu objeto de estudo principal não consistia, de fato, no "ambiente virtual de aprendizagem”. Sendo assim, este artigo consolida a análise de 50 teses sobre o referido tema. É importante salientar que os documentos referentes ao ano 2013 ainda estavam em fase de inclusão na base de dados. Portanto eles não constituíram dados definitivos para esta análise. O total de teses (n) ${ }^{3}$ analisadas garantiu uma amostra representativa da base de dados ${ }^{4}$. O total de teses consideradas para a análise e registradas na série histórica, por ano, está representado na Figura 1:

Número de teses por ano - entre 2003 e 2012

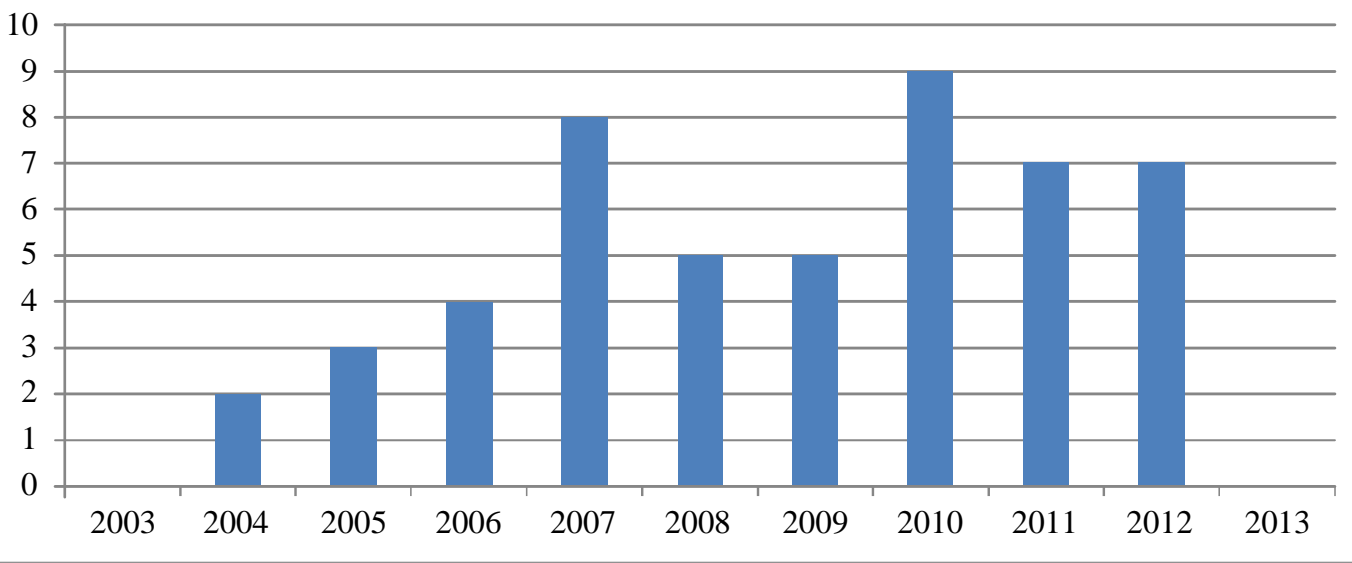

Figura 1 - Número de teses disponíveis na BDTD, no período entre 2003 e 2012.

Para realizar a análise de conteúdo dos documentos, os dados foram compilados em planilha eletrônica e em arquivo de texto. Os principais dados reunidos na planilha eletrônica foram: o ano da defesa, instituição, orientador(a), área de estudo ou Programa

\footnotetext{
${ }^{1}$ Endereço eletrônico: http://bdtd.ibict.br.

${ }^{2}$ A coleta de dados foi realizada no período entre agosto e novembro de 2013.

${ }^{3}$ Sendo que a notação "n” corresponde ao número de trabalhos encontrados na amostra analisada.

${ }^{4}$ A amostra analisada representa 5\% de erro amostral, com nível de confiança entre $90 \%$ e 99\% - sendo $n=48$, para $90 \%$ e $n=54$, para $99 \%$.
} 
de Pós-graduação (PPG), palavras-chave, objetivo geral, método da pesquisa, AVA do estudo e categorias foco do estudo (a partir da análise das palavras-chave e resumo de cada uma das teses). As palavras-chave coletadas foram reunidas em um arquivo de texto para proceder à análise das palavras ali contidas.

A tese como fonte de pesquisa constitui-se em um corpus de documento. Para Franco (2005, p. 49), citando Bardin, corpus "é o conjunto de documentos considerados para serem submetidos aos procedimentos analíticos”. A partir desta definição, a partir de leitura crítica, foi realizada a análise de conteúdo do corpus das teses. Essa análise permitiu identificar características do texto que permitem explorar o tema. Os dados coletados foram tabulados, codificados, apresentados em percentuais e representados por elementos visuais, na forma de gráficos e tabelas. Para complementar a análise de frequência das diferentes categorias, foi realizada a verificação da ocorrência de palavras nas palavras-chave, elaborando nuvens de palavras com o uso do aplicativo web TagCrowd, possibilitando a visualização de frequência com que as palavras aparecem em um texto, como forma de análise qualitativa (STEINBOCK, 2012). Tal técnica é denominada análise de conteúdo (TRIVIÑOS, 2011), enquanto perspectiva de análise alternativa à tradicional, tratando "da sistematização, da tentativa de conferir maior objetividade a uma atitude que conta com exemplos dispersos, mas variados, de pesquisa com textos” (ROCHA, DEUSDARÁ, 2005, p. 308).

\section{Resultados e Discussão}

Nesta seção são apresentados: o número de teses por instituições, as áreas temáticas dos estudos, as palavras-chave utilizadas nas teses, os métodos de pesquisa empregados nos trabalhos, AVA utilizados como contexto ou objeto da tese e a correspondência às categorias propostas por Roque et al. (2001).

Na Figura 2 é possível visualizar o número de teses por instituição (por ano), sendo a Universidade Federal do Rio Grande do Sul (UFRGS) a instituição com maior contribuição na temática $(n=25)$.

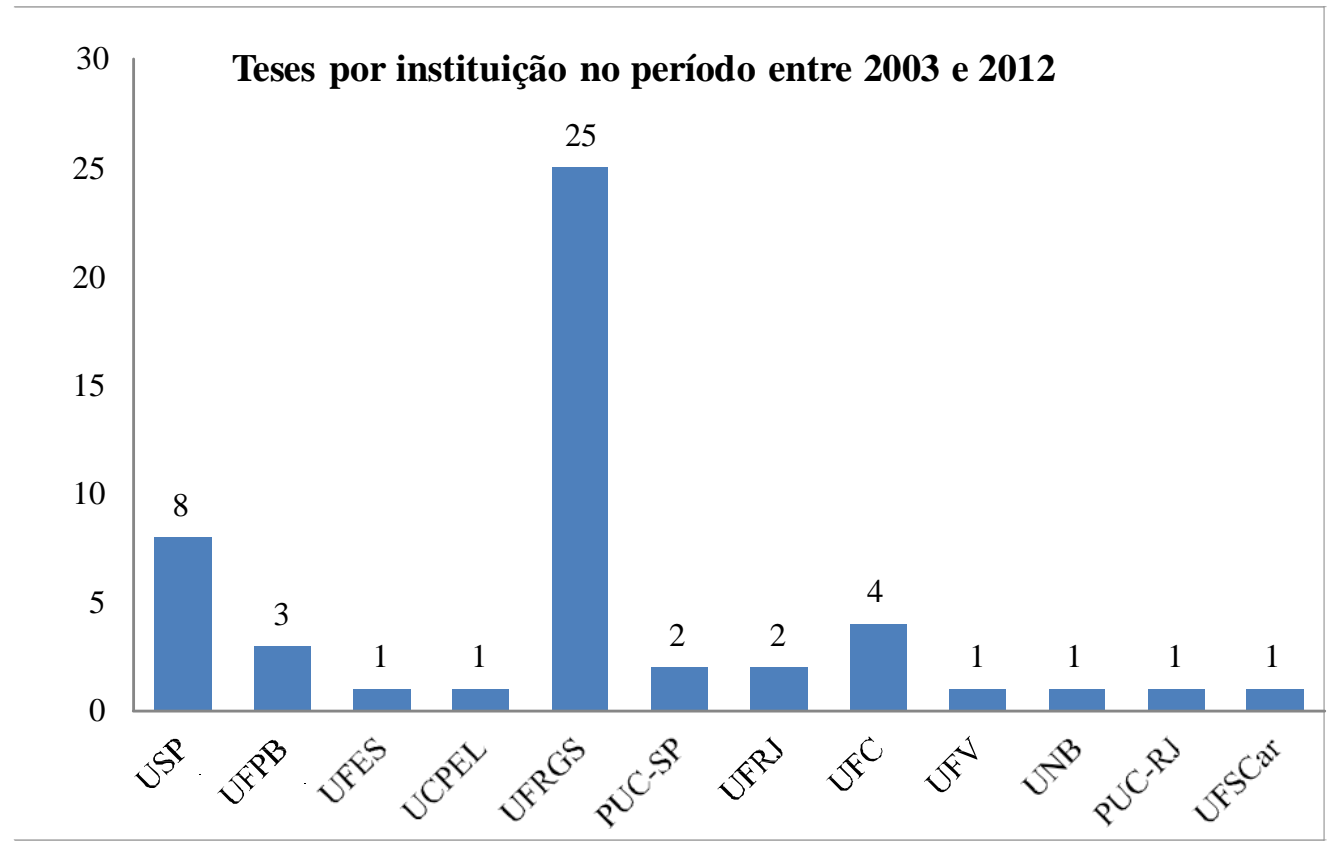

Figura 2 - Teses por instituição no período entre 2003 e 2012. 
Quanto às áreas temáticas, a maioria das teses é oriunda da Informática na Educação com 17 publicações (34\%). A segunda maior área é a Educação, com 13 teses (26\%). Depois aparecem a Enfermagem com 4 teses (8\%) e a Linguística e Letras ambas com duas teses (4\%). Outras áreas menos frequentes também podem ser visualizadas na Tabela 1:

Tabela 1 - Áreas de estudo mais frequentes.

\begin{tabular}{c|c|c}
\hline Área de estudo & $\mathbf{n}$ & $\mathbf{\%}$ \\
\hline Educação & 13 & 26 \\
\hline Informática na Educação & 17 & 34 \\
\hline Enfermagem & 4 & 8 \\
\hline Letras & 2 & 4 \\
\hline Linguística & 2 & 4 \\
\hline Outros & 12 & 24 \\
\hline Total & 50 & 100 \\
\hline
\end{tabular}

Analisando-se as palavras-chave do conjunto de teses e que resultou na Tagcloud (Figura 3) contendo 50 palavras, observou-se as seguintes ocorrências: educação $(n=29)$, aprendizagem $(n=27)$, ensino $(n=24)$, distância $(n=20)$, e ambiente $(n=17)$. Observa-se que, apesar das palavras encontradas serem bastante amplas, estas referem-se predominamente a questões pedagógicas em detrimento de questões tecnológicas ou administrativas.

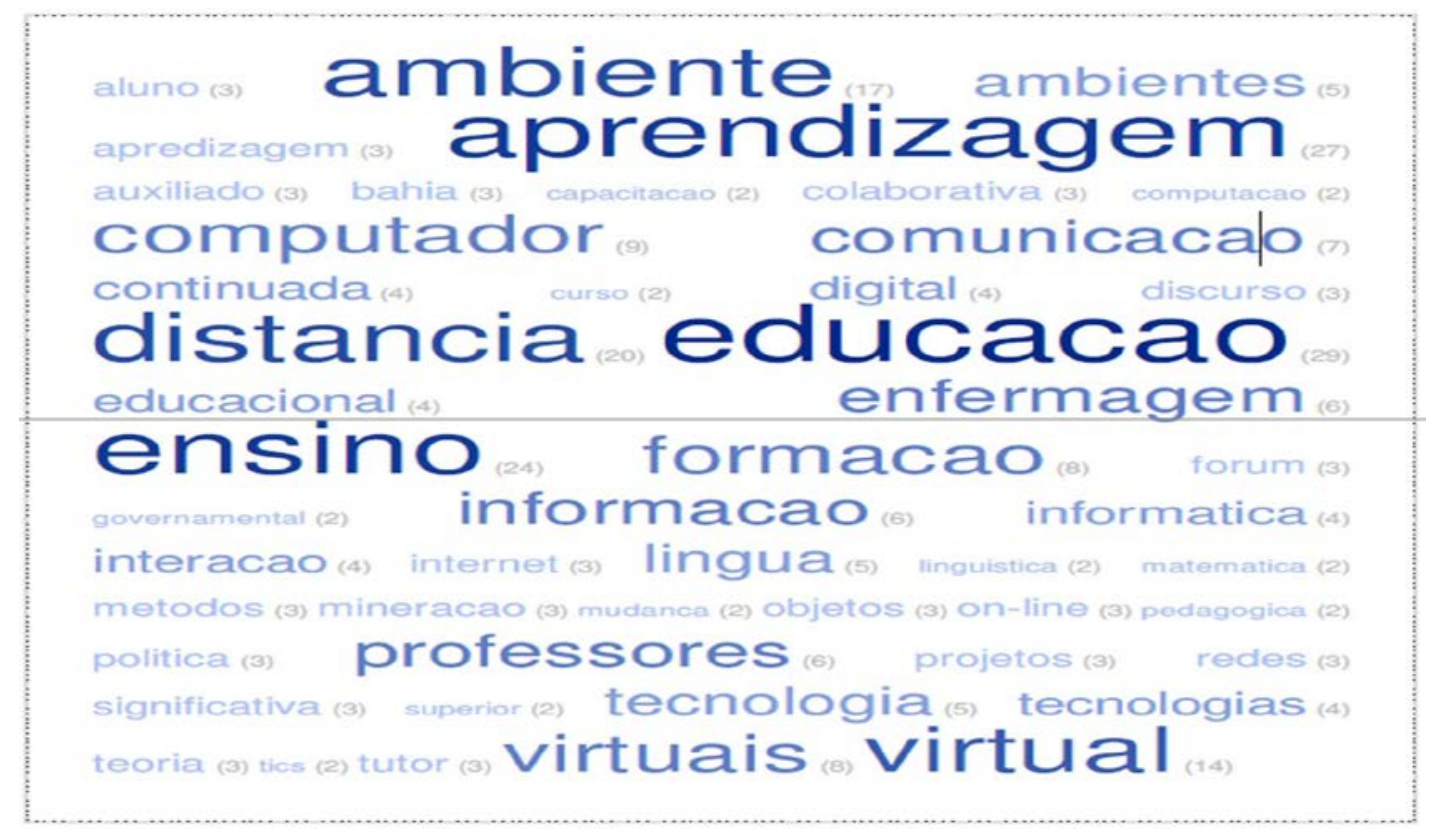

Figura 3 - Tagcloud contendo 50 palavras, elaborada com o aplicativo TagCrowd.

Após analisar os métodos de pesquisa empregados nas teses (Figura 4), foi possível concluir que a maioria utilizou o método de Estudo de caso, totalizando 64\% 
$(\mathrm{n}=32)$, sendo que os demais utilizaram os métodos Experimental e Survey, sendo respectivamente $28 \%(\mathrm{n}=14)$ e $8 \%(\mathrm{n}=4)$ dos trabalhos.

\section{Método de pesquisa empregado pelas teses}

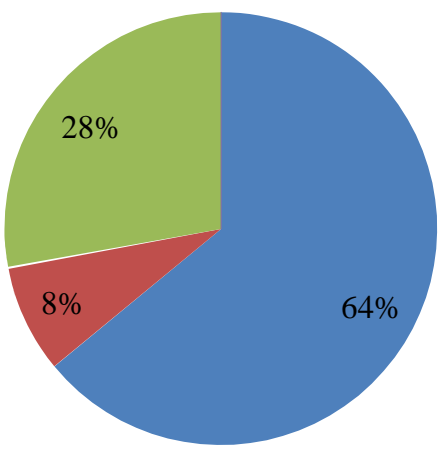

$$
\begin{aligned}
& \text { Estudo de caso } \\
& \text { Survey } \\
& \text { Experimental }
\end{aligned}
$$

Figura 4 - Método de pesquisa empregado pelas teses.

Na Figura 5 são demonstrados os AVA como contexto ou objeto da tese, sendo que $20 \%(n=10)$ utilizaram o Moodle, 14\% $(n=7)$ utilizaram o TelEduc, $10 \%(n=5)$ utilizaram o Rooda, 4\% ( $\mathrm{n}=2)$ utilizaram o Amadis, 36\% ( $\mathrm{n}=18)$ utilizaram outros ambientes, diferentes dos já citados e 16\% $(\mathrm{n}=8)$ não mencionaram o ambiente utilizado.

\section{AVA utilizados como contexto para as teses}

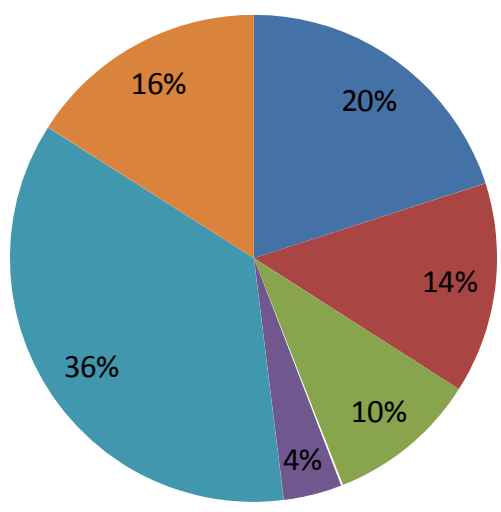

Moodle

- TelEduc

- Rooda

Amadis

- Outros

Não menciona

Figura 5 - AVA utilizados como contexto para as teses.

A Figura 6 apresenta, as teses de acordo com as categorias propostas por Roque et al. (2001). Essas categorias compreendem Interface, Navegação, Avaliação, Recursos Didáticos, Comunicação/Interação, Coordenação e Apoio Administrativo. 


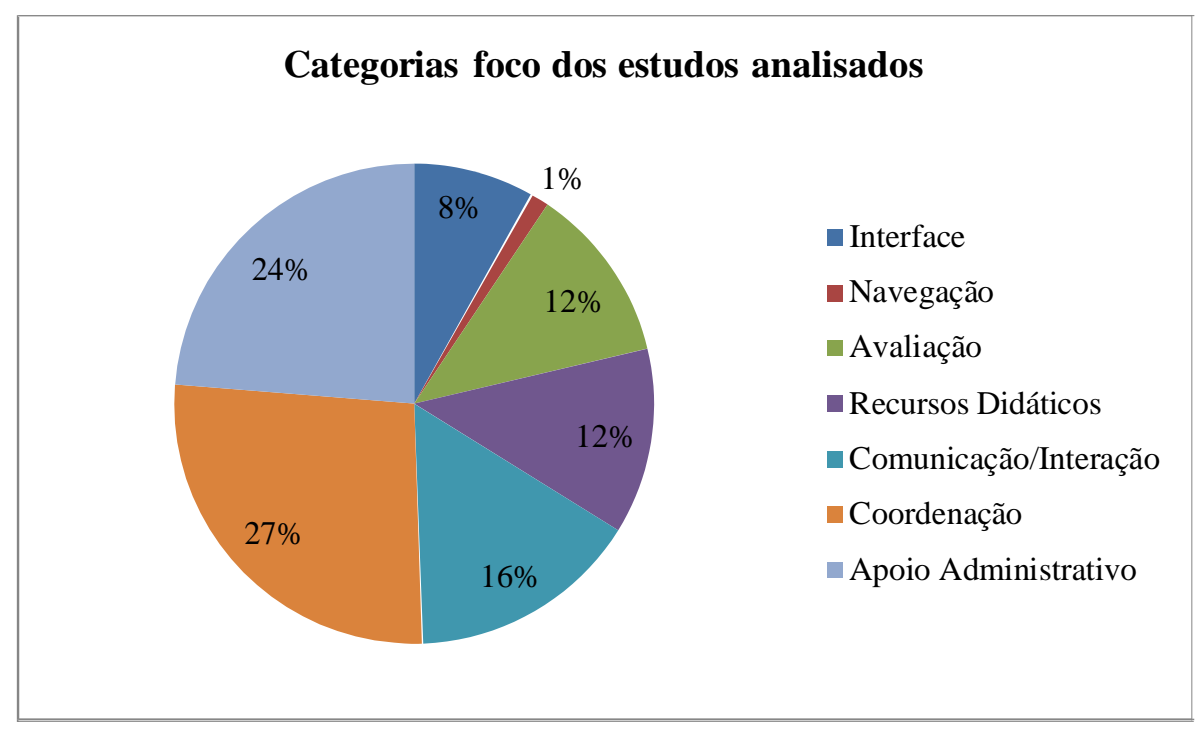

Figura 6 - Categorias foco das teses.

A partir da análise das teses, percebeu-se que 27\% (n=43) dos trabalhos tiveram como foco a categoria Coordenação, 24\% (n=38) a categoria Apoio Administrativo, $16 \%(n=25)$ a categoria Comunicação e Interação, 12\% $(n=19)$ a categoria Avaliação, $12 \%(n=20)$ a categoria Recursos Didáticos, $8 \%(n=13)$ a categoria Interface e apenas $1 \%(n=2)$ utilizou como foco de estudo apenas a categoria Navegação. A análise revelou que três teses abordaram somente categorias pontuais, indicadas por Roque et al. (2001):

A pesquisa de Nunes (2012) buscou analisar o processo de avaliação da tutoria a distância concebido para o curso de Pós-Graduação em Informática na Educação do Instituto Federal do Espírito Santo (IFES) e refletir sobre mudanças de condutas ocorridas. A tese, que consistiu em um estudo de caso, utilizando o AVA Moodle como contexto, com foco na categoria Avaliação, foi apresentada ao PPG em Educação da Universidade Federal do Espírito Santo.

A pesquisa de Santos (2009) buscou verificar de que maneira a inserção de um agente conversacional em um AVA pode interferir na carga cognitiva dos materiais educacionais e influenciar no desempenho e engajamento dos estudantes, no contexto do ensino à distância. A tese, que consistiu em um experimento, utilizando o AVA Moodle como contexto, também com foco na categoria Coordenação, foi apresentada ao PPG em Informática na Educação da Universidade Federal do Rio Grande do Sul.

Já a pesquisa de Silva (2012), buscou analisar a participação do enfermeiro no desenvolvimento, acompanhamento e supervisão de alunos de Enfermagem em estágio curricular por meio de uma estratégia de intervenção educativa a distância. A tese, que consistiu em um estudo de caso, utilizando o AVA Moodle como contexto, com foco na categoria Coordenação, foi apresentada ao PPG em Enfermagem da Universidade Federal do Rio Grande do Sul.

A análise também revelou que somente uma, das 50 teses analisadas, contemplou todas as categorias indicadas por Roque et al. (2001). A pesquisa de Aquino (2010) buscou verificar os efeitos da utilização de uma tecnologia educativa na aprendizagem de acadêmicos de enfermagem com relação ao conteúdo de contracepção. A tese, de natureza experimental, que utilizou o AVA SOLAR ${ }^{5}$, foi apresentada ao PPG em Enfermagem da Universidade Federal do Ceará.

\footnotetext{
${ }^{5}$ Ambiente criado pelo Instituto UFC Virtual.
} 


\section{Conclusões}

O método da revisão integrativa foi utilizado para análise das teses sobre AVA na produção científica brasileira de 2003 a 2012, disponibilizada no repositório da BDTD. Foi possível perceber o comportamento histórico e que há o interesse crescente da comunidade acadêmica para o desenvolvimento de pesquisas relacionadas aos AVA em diferentes universidades brasileiras.

As restrições do estudo apontam para o acesso às teses de 2013, que não puderam ser computadas e de eventuais limitações e oscilações da base de dados para a extração das informações, portanto entende-se que parte das teses sobre o tema foi analisada. Ressalta-se que os dados obtidos foram checados e validados duas vezes pelos pesquisadores, para evitar a análise e divulgação de resultados inconsistentes.

Esta revisão aponta ainda para oportunidades de pesquisa, a partir da análise dos dados levantados e contribui para que se possa compreender o cenário da pesquisa científica brasileira dos estudos sobre os AVA no período. Tendo em vista o panorama apresentado do desenvolvimento científico de estudos sobre AVA, sugerem-se estudos, inspirados em Gonzales (2005), que poderão nortear futuras pesquisas sobre o tema:

- Sugestão 1 - estudos com foco na funcionalidade de produção dos alunos no AVA: poderão investigar o desenvolvimento da análise e mineração de dados no AVA, o desenvolvimento de ontologias para mapear o conhecimento compartilhado no ambiente e o incremento da utilização de componentes de inteligência artificial, como os agentes inteligentes, por exemplo.

- Sugestão 2 - estudos com foco na funcionalidade de coordenação e comunicação dos AVA: poderão investigar a inclusão e disseminação de tecnologias assistivas no AVA, a avaliação de aprendizagem e impactos da tecnologia nos alunos, docentes e gestores de EaD, por exemplo.

- Sugestão 3 - estudos com foco na funcionalidade de cooperação e administração dos AVA: poderão investigar os possíveis papéis do AVA na gestão de cursos em EaD, envolvendo questões de planejamento e acompanhamento pelas instituições de ensino e gestores da modalidade, por exemplo.

Ao final, ressalta-se que, a maioria das teses analisadas tratou de aspectos pontuais dos AVA, que sem dúvida, são relevantes, mas insuficientes para a compreensão do assunto na sua totalidade, pois o tema necessita ser pesquisado em suas diversas dimensões e considerando sua complexidade. As sugestões apresentadas por este estudo, ainda que em formato de indicações macro, poderão auxiliar no desenvolvimento de novas pesquisas, especialmente de cunho interdisciplinar.

\section{Referências}

AQUINO, P. S. Tecnologia educativa no ensino de enfermagem em contracepção. Fortaleza: PPG em Enfermagem, 2010. 101p. Tese de Doutorado.

BACH, T. M.; DOMINGUES, M. J. C. S.; WALTER, S. A. Tecnologias da informação e comunicação no ensino: um estudo bibliométrico e sociométrico de 1997-2011. Avaliação, Sorocaba, v. 18, n. 2, p. 393-416, jul. 2013.

BOTELHO, L. L., CUNHA, C. C. A., MACEDO, M. O método da revisão integrativa nos estudos organizacionais. Gestão e Sociedade. Belo Horizonte, v. 5, n. 11, Maio/Agosto, 2011. 
BRITO, L. M.; JUNIOR, R. G.; GOMES, I. G. S.; MOTA, J. B. Ambientes virtuais de aprendizagem como ferramentas de apoio em cursos presenciais e a Distância. CINTED-UFRGS. Novas Tecnologias na Educação. Porto Alegre, v. 11, n. 1, Julho, 2013.

CARDOSO, A. L. M. S.; BURNHAM, T. F. Ambientes virtuais de aprendizagem alternativos e inovadores para potencializar novas soluções em EaD. CINTED-UFRGS. Novas Tecnologias na Educação. Porto Alegre, v. 9, n. 1, Julho, 2011.

COUTINHO, L. Aprendizagem on-line por meio de estruturas de cursos. In: Educação a distância: o estado da arte. LITTO, F., FORMIGA, M. (orgs). São Paulo: Pearson Education do Brasil, 2009.

FRANCO, M. L. P. B. Análise de conteúdo. 2. ed. Brasília: Líber Livro, 2005. GIL, A. C. Métodos e técnicas de pesquisa social. 6. ed. São Paulo: Atlas, 2008. GONZALES, M. Fundamentos da tutoria em educação a distância. São Paulo: Avercamp, 2005.

JACKSON. G. B. Methods for integrative reviews. Review of Educational Research Fall, v. 50, n. 3, pp. 438-46, 1980.

MENDES, K. D. S., SILVEIRA, R. C. C. P., GALVÃO, C. M. Revisão integrativa: método de pesquisa para a incorporação de evidências na saúde e na enfermagem.

Texto Contexto Enferm, v. 17, n. 4, p. 758-64, 2008.

MOORE, M. G.; KEARSLEY, G. Educação a distância: uma visão integrada - Edição Especial ABED. São Paulo: Thomson Learning, 2007.

NUNES, V. B. Processo avaliativo de tutores a distância em um curso de PósGraduação e reflexões sobre mudanças de conduta. Vitória: PPG em Educação, 2012. 379p. Tese de Doutorado.

ROCHA, D.; DEUSDARÁ, B. Análise de conteúdo e análise do discurso: aproximações e afastamentos na (re)construção de uma trajetória. Alea, v. 7, n. 2, p. 305-322, 2005.

ROQUE, G. O. B., CHAMOVITZ, I.; CAMPOS, J. A. S.; ARAÚJO, J. F. S.; GOUVEA, M. T.; CARDOSO, R. P.; AZAMBUJA, S.; MOURA, S. A. Aspectos relevantes para o desenvolvimento de ambientes educacionais para a web. In: III Conferência Iberoamericana en Sistemas, Cibernética e Informática (CISCI). Anais... Miami: CISCI, 2004.

SANTOS, L. M. A. A inserção de um agente conversacional animado em um ambiente virtual de aprendizagem a partir da teoria da carga cognitiva. Porto Alegre: PPG em Informática na Educação, 2009. 125p. Tese de Doutorado.

SILVA, A. P. S. S. A enfermeira e a corresponsabilidade pela formação do aluno em estágio curricular. Porto Alegre: PPG em Enfermagem, 2012. 115p. Tese de Doutorado.

STEINBOK, D. What is tagCrowd? Disponível em: <http://www.tagcrowd.com>. Acesso em 02 nov. 2014.

TRIVIÑOS, A. N. S. Introdução à pesquisa em ciências sociais: a pesquisa qualitativa em educação. 1. ed. 20 reimpr. São Paulo: Atlas, 2011.

WHITTEMORE R., KNAFL, K. The integrative review: updated methodology. Journal of Advanced Nursing, v. 52, n. 5, p. 546-553. 2005.

ZAWACKI-RICHTER, O.; BÄCKER, E. M.; VOGT, S. Review of distance education research (2000 to 2008): analysis of research areas, methods, and authorship patterns. International Review of Research in Open and Distance Learning, v. 10, n. 6, dez. 2009. 